\title{
Quadtree Coding with Adaptive Scanning Order for Space-borne Image Compression ${ }^{\text {th }}$
}

\author{
Hui Liu ${ }^{a}$, Ke-Kun Huang ${ }^{\mathrm{a}, *}$, Chuan-Xian Ren ${ }^{\mathrm{b}}$, Yu-Feng Yub ${ }^{\mathrm{b}}$, Zhao-Rong Laic \\ ${ }^{a}$ School of Mathematics, JiaYing University, Meizhou, Guangdong, 514015, China. \\ ${ }^{b}$ Intelligent Data Center and Department of Mathematics, Sun Yat-Sen University, Guangzhou 510275, China. \\ ${ }^{c}$ Department of Mathematics, College of Information Science and Technology, JiNan University, Guangzhou 510632, China.
}

\begin{abstract}
Space-borne equipments produce very big images while their capacities of storage, calculation and transmission are limited, so low-complexity image compression algorithms are necessary. In this paper, we develop an efficient image compression algorithm based on quadtree in wavelet domain for this mission. First, we propose an adaptive scanning order for quadtree, which traverses prior the neighbors of previous significant nodes from bottom to the top of quadtree, so that more significant coefficients are encoded at a specified bit rate. Second, we divide the entire wavelet image to several blocks and encode them individually. Because the distortion-rate usually decreases as the level of the quadtree increases with the adaptive scanning order, to control bit rate for each block, we set the points exactly after coding each level of the quadtree as the candidate truncation points. The proposed method can provide quality, position and resolution scalability, which is simple and fast without any entropy coding, so it is very suitable for space-borne equipments. Experimental results show that it attains better performance compared with some stateof-the-art algorithms.
\end{abstract}

Keywords: Remote sensing image compression, quadtree coding, rate-distortion optimization, adaptive scanning order

\section{Introduction}

With the rapid development of sensor technology on space-borne equipments, high spatial resolution remote sensing images are becoming more easily acquired. Since the images will take up a great deal of storage space, and the hardware of space-borne equipments is limited, great efforts are made to seek low-complexity image compression algorithms. The main requirements to image compression techniques for a considered application are two folds. First, the distortion between original image and decoding image needs as small as possi-

\footnotetext{
This work is supported in part by National Science Foundation of China under Grants 61403164 and 61572536, in part by the Fundamental Research Funds for the Central Universities under Grant 16lgzd16, and in part by the Foundation for Distinguished Young Talents in Higher Education of Guangdong, China(2013LYM_0085).

${ }^{*}$ Corresponding author

Email addresses: imlhxm@163.com (Hui Liu), kkcocoon@163. com (Ke-Kun Huang),

rchuanx@mail.sysu.edu.cn (Chuan-Xian Ren), yuyufeng220@163.com (Yu-Feng Yu),

laizhrejnu.edu.cn (Zhao-Rong Lai)
}

ble at a certain specified compression ratio. Second, the algorithm's complexity needs as low as possible.

Because of the high energy compaction property of wavelet transform, most image compression algorithms are based on set partition. A class of successful methods exploit the property of self-similarity across scales in wavelet image, such as Embedded Zerotree Wavelet (EZW) [1], Set Partitioning in Hierarchical Tree (SPIHT) [2] and Set Partitioned Embedded Block (SPECK) [3]. These methods construct zero-tree through spatial similarity of the wavelet coefficients at different scales in each bit-plane so that a large number of non-significant coefficients are predicted successfully by a root of zero-tree.

Other than the zero-tree methods, some methods utilize the clustering characteristic within each subband. The Quadtree Coding (QC) is a successful method that takes the advantage, and has been reported in some literatures $[4,5,6]$. QC is based on hierarchical set partitioning. There is only one node at the top level of quadtree, which consists of all coefficients. If there is any significant coefficient in a node, then it splits into $2 \times 2$ de-

March 18, 2017 
scendent nodes that are two dimensional homogeneous 86 blocks. We can perform the splitting recursively to ${ }_{87}$ quickly locate a significant coefficient. Given the splitting information, the same significance map can be reproduced by the decoder. Through the bit-plane coding, QC produces a lossless and embedded data stream, supports quality scalability, and permits region-of-interest coding. The excellent performance for this scheme is presented in [6].

In recent years, some compression schemes that are specifically designed for remote sensing images have been proposed. The Consultative Committee for Space Data Systems (CCSDS) published an Image Data Compression (CCSDS-IDC) [7], which specifically targets space-borne equipments, and focuses more on compression and less on options of handling and distributing compressed data. There are some extensions have been presented for the CCSDS-IDC [8][9]. JPEG2000 [10] is a standard image compression algorithm based on wavelet transform, and it is also extended for spaceborne equipments. In [11], the wavelet transform in JPEG2000 is replaced by a two-dimensional oriented wavelet transform and it outperforms JPEG2000 for remote sensing images. Kulkarni et al. [12] presented a scan-based method that can use JPEG2000 with incrementally acquired data. However, some components of JPEG2000 that help to provide high compression performance also have high implementation complexity, which limits JPEG2000 to be a standard for airborne mission.

Besides JPEG2000 and CCSDS-IDC, the other compression algorithms are also applied for remote sensing image. In [13], a modified listless strip based SPIHT is proposed to reduce system complexity and minimize processing time and memory usage. In [14], an improved SPIHT is proposed for multispectral image compression for various band images with high resolution. 120 In [15] and [16], two improved compressions of mul- 121 tispectral images based on classified transform coding 122 are proposed, which are superior to that of the origi- 123 nal class-based multispectral image coder [17]. In [18], 124 the lapped transform and Tucker decomposition are proposed for hyperspectral image compression. In [19], a compression algorithm of hyperspectral remote sens- ${ }^{125}$ ing images is proposed, which introduces a tensor decomposition technology to approximately decompose the original tensor data into a core tensor multiplied by a factor matrix. In [20], a new efficient region-based scheme for the compression of multispectral remote- 129 sensing image is proposed, which outperforms some 130 state-of-the-art methods. In [21], an overview of several 131 standards for remote sensing data compression is pro- 132 vided, discussing both mono-band and multi-band compression, and lossless, lossy and near-lossless compression. In [22], a novel wavelet-based scheme to increase coefficient independence in hyperspectral images is introduced for lossless coding, which uses multivariate regression to exploit the relationships among wavelet transformed components, and outperforms some most recent coding methods.

The tree-based partitioning methods are also applied for space-borne equipments. In [23], a SAR complex image data compression algorithm based on QC in wavelet transform domain is proposed, showing that QC achieves the best performance for SAR complex image compression. In [24], the quadtree in QC is replaced by the binary tree, which achieves better performance. In [25], a Human vision-based Adaptive Scanning (HAS) for the compression of remote sensing image is proposed, which generates an importance weighting mask according to the human visual characteristics. In [26], a content-based adaptive scanning scheme is proposed for remote sensing image compression, which provides different scanning orders among and within subbands.

However, the performance of QC is not good enough. We find that the neighbors of the previous significant coefficients are more likely significant at current bitplane, so we develop an adaptive scanning order for quadtree to achieve higher compression ratio. Moreover, QC needs a lot of memory to store the quadtree tree for large-scale images, and does not provide random access property, so we divide the entire wavelet image to several blocks and encode them individually, then apply rate-distortion optimization to achieve higher performance.

Our main contributions can be listed as follows:

- We propose an adaptive scanning order for quadtree, which traverses prior the neighbors of previous significant nodes from bottom to the top of quadtree, so that more significant coefficients are encoded at a specified bit rate.

- We divide the entire wavelet image to several blocks and encode them individually, then apply rate-distortion optimization to achieve higher performance.

The remainder of the paper is organized as follows: In section 2, we describe the proposed method in detail. The experimental results are given in section 3. Finally, the conclusion is provided in section 4 . 


\section{The proposed method}

\subsection{Quadtree coding}

The Quadtree Coding (QC) takes the advantage of the clustering characteristic within each subband, and has been reported in some literatures $[4,5,6]$. The core of this approach is based on hierarchical set partitioning. ${ }^{178}$ The bottom level of the quadtree consists of all coeffi- 179 cients. Each node of the next higher level is then set to 180 the maximum value of its four child nodes. In Figure 1, 181 there is a $4 \times 4$ wavelet image, and the bottom level of the 182 quadtree consists of 16 nodes. The higher level consists ${ }^{183}$ of 4 nodes, where the first red node (9) is the maximum 184 of the four red coefficients at the bottom level $(9,5,4,3)$. 185

We provide the notation for the quadtree tree as fol- 186 lows. Suppose there is a wavelet image with size of $L_{0}=187$ $2^{N} \times 2^{N}$, which is transformed into one-dimensional vec- 188 tor using Morton scanning order, denoted by $I(t)$ for 189 $t=1,2, \cdots, L_{0}$.

From bottom to the top, we construct the quadtree 191 $\Psi(t)$ for $1 \leq t \leq L_{0}$. The number of nodes at the bottom 192 level of the quadtree is equal to $L_{0}$, and the number of 193 nodes at the next upper level is equal to $\frac{L_{0}}{4}$, and so on. ${ }^{194}$ The depth of the quadtree is $\frac{\log \left(L_{0}\right)}{\log (4)}=N$, and the total 195 number of nodes of the quadtree is $L=\sum_{d=0}^{N} 4^{d}$.

The bottom level of the quadtree consists of all the ${ }^{197}$ coefficients:

$$
\Psi(t)=I\left(t-\left(L-L_{0}\right)\right) \text { for } L-L_{0}+1 \leq t \leq L .
$$

The upper levels of the tree are defined iteratively:

$\Psi(t)=\max \{|\Psi(4 t-2)|,|\Psi(4 t-1)|,|\Psi(4 t)|,|\Psi(4 t+1)|\} \quad \begin{aligned} & 203 \\ & 204\end{aligned}$ for $1 \leq t \leq L-L_{0}$.

(2) 205

After constructing the quadtree, we can traverse the ${ }^{206}$ tree by depth-first. For each bit plane, going from top to ${ }^{207}$ the bottom level of the quadtree, if a node is insignificant with respect to the current threshold, then it is coded by " 0 ", otherwise it is coded by " 1 ", and the process is recursively applied to the four children. If the process reaches the bottom level of the tree and the corresponding node is significant, then the sign of the coefficient is coded. We can neglect some codes as follows:

- If one node is a previous significant node, then this node must be significant with the current threshold.

- If one node is significant and its three children are insignificant, then the last child must be significant

The process can be expressed as a function code $=219$ $Q C\left(\Psi, t, T_{k}\right)$ as Algorithm 1, where $t$ is a tree node in- 220 dex of the quadtree, and $T_{k}$ is a threshold where $T_{0}=221$ $2^{\left\lfloor\log _{2} \Psi(1)\right\rfloor}$ and $T_{k}=T_{0} / 2^{k}$. The algorithm uses a stack to avoid recursive function.

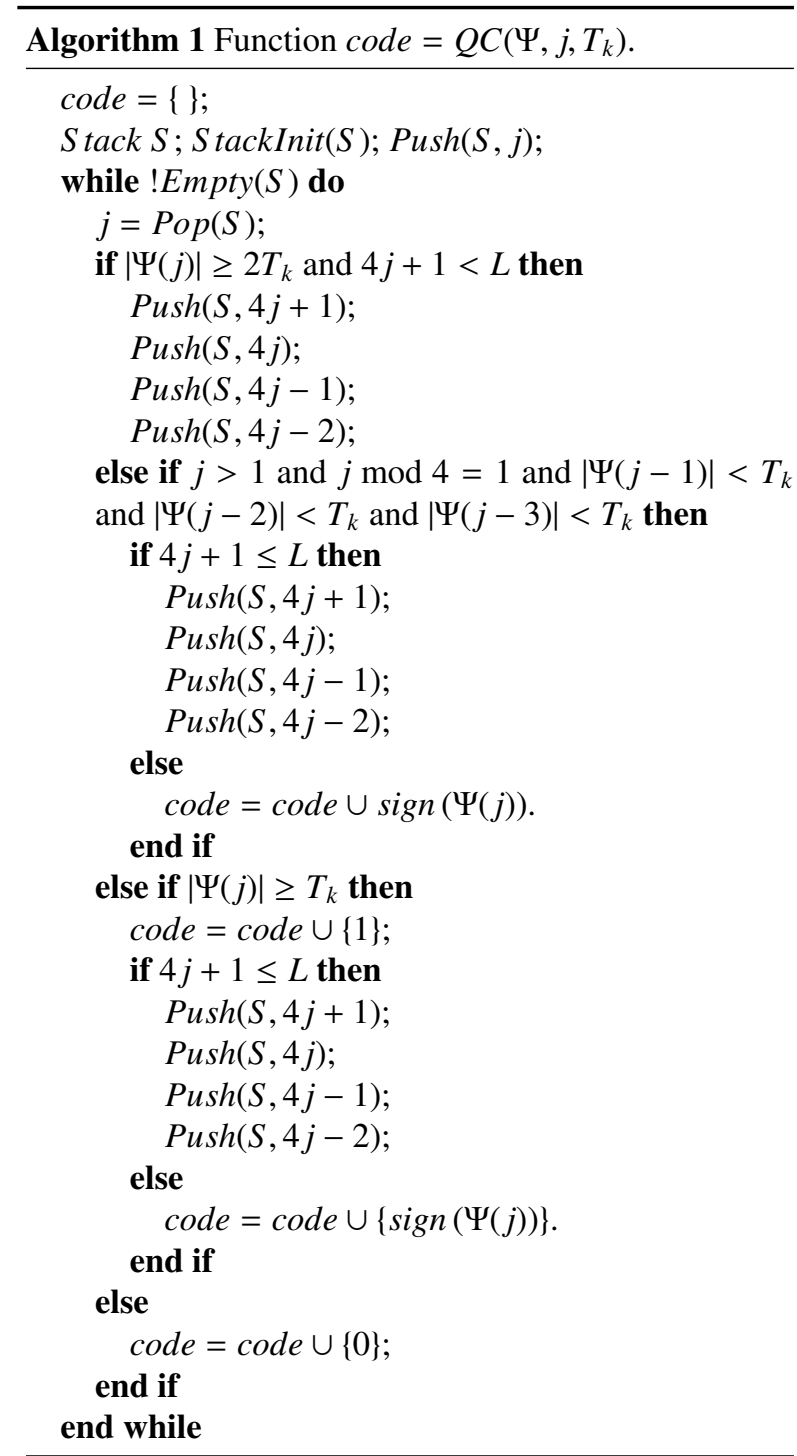

\subsection{Adaptive scanning coding}

In quadtree, if a node has a significant parent with larger threshold, in other words, some brothers of the node are significant previously, we find that this node is very likely to be significant with the current threshold. Moreover, the lower the level of the quadtree is, the higher the probability to be significant is. Figure 2 gives an example. We construct a quadtree for a block of a wavelet image. We first find the nodes whose absolute values are greater than the threshold 64 . With the threshold 32, for each node at the bottom level of the tree, if there are some previous significant brothers 


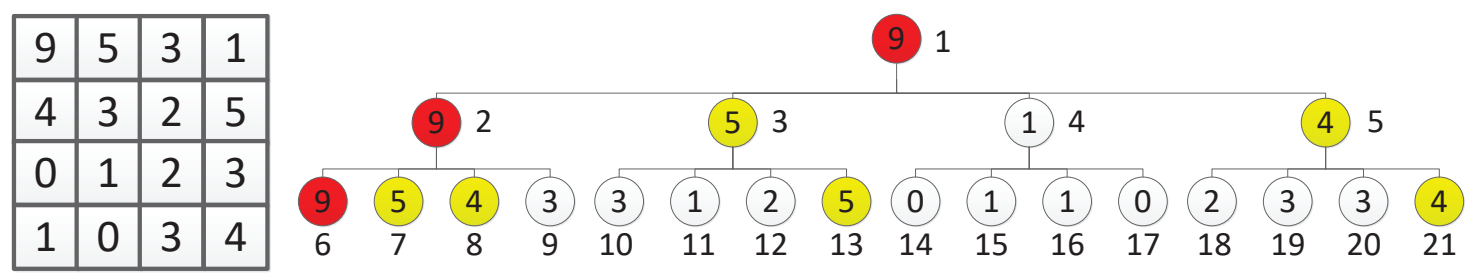

Figure 1: A simple example to illustrate the proposed method. Left: a block to be encoded. Right: The corresponding quadtree. The number in the tree node indicates the value of corresponding coefficient, and the number on the right of the tree node denotes the index of the node.

whose absolute values are greater than 64, then the node is collected to be traversed first. We find that 31 percent of these coefficients are significant with the threshold 32 , i.e., the significance probability is $31 \%$. At the higher levels of the tree, the significance probabilities are $27 \%, 20 \%$ and $14 \%$, respectively. With the threshold 16 , the significance probability decreases as the level of the tree increases too. Because at the higher levels of the tree, the leafs of the sub-trees rooted at the brothers of ${ }_{249}^{248}$ significant nodes are farther from the significant coefficients, so the significance probability decreases and we ${ }_{251}$ can traverse the quadtree from bottom to the top level.

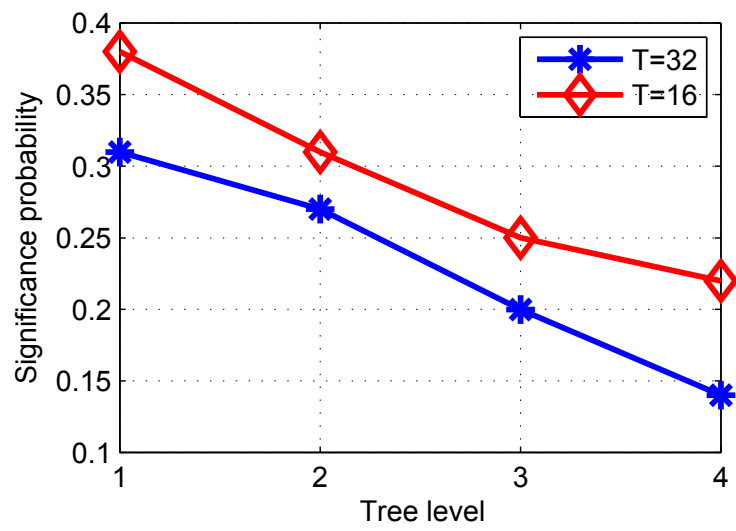

Figure 2: The significance probability versus tree level With each threshold, the significance probability decreases as the level of the tree increases.

In other words, we propose to scan the neighbors of the previous significant coefficients before other regions are scanned. The scanning order are adaptively determined by the previous significant nodes. The detail steps of the proposed method can be described as a ${ }_{274}$ function code $=Q C A\left(\Psi, T_{k}\right)$ as Algorithm 2.

Algorithm 2 Function code $=Q C A\left(\Psi, T_{k}\right)$.

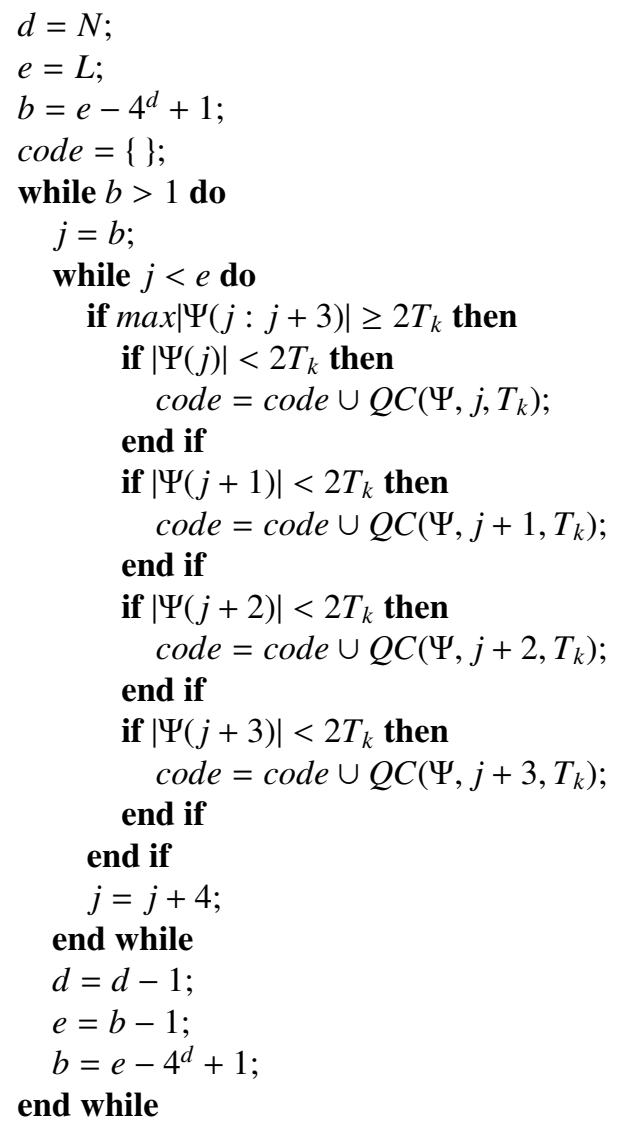

Figure 1 gives the adaptive scanning order for the quadtree of a $4 \times 4$ block. Suppose the red nodes and the yellow ones are the previous significant nodes whose values are no less than 4 . Then with the threshold $T=2$, the adaptive scanning order is defined as follows. At the bottom level, the $9^{\text {th }}, 10^{\text {th }}, 11^{\text {th }}, 12^{\text {th }}, 18^{\text {th }}, 19^{\text {th }}, 20^{\text {th }}$ nodes are scanned first, because their brothers contain some previous significant nodes, while the other nodes at the bottom level should not be scanned at this time. 
At the next upper level, the subtree rooted with the $4^{\text {th }}$ node is encoded.

\subsection{Block coding and optimization}

If we directly use QCA to encode the entire wavelet image, then it needs a lot of memory to store the quadtree and does not provide random access property. We can divide the wavelet image into several blocks and encode them by QCA independently. To control bit rate for each block, we can optimize the distortion-rate for each block. That is, we first select some coding stages at each block as the valid truncation points, such that the corresponding local distortion-rates are strictly decreasing for each block. Then we select a final truncation point for each block such that the rate reaches the specified value and the distortion-rate is maximized.

In the process of the distortion-rate optimization $[10,27,28]$, how to select the truncation points is a key problem. We find that with the adaptive scanning order, the distortion-rate usually decreases as the level of the quadtree increases, so we can set the points exactly after coding each level as the candidate truncation points.

Let

$$
S_{j}=\frac{D_{j-1}-D_{j}}{R_{j}-R_{j-1}}
$$

denote the local distortion-rate for a block, where $R_{j}$ is the bit rate and $D_{j}$ is the corresponding distortion at $j^{\text {th }}$ candidate truncation point. Figure 3 shows distortionrates of the candidate truncation points of when using the proposed method to encode a block with size of $2^{6} \times$ $2^{6}$.

We select some of the candidate truncation points of the independent block such that the distortion is minimized at a specified bit rate. If $S_{j+1}>S_{j}, j^{\text {th }}$ candidate truncation point cannot be selected, and we need to update

$$
S_{j+1}=\frac{D_{j-1}-D_{j+1}}{R_{j+1}-R_{j-1}}
$$

and compare it with $S_{j-1}$. This process is performed until $S_{j+1}<S_{j_{k}}$. We can select the strictly decreasing $S_{j_{k}}$ as a series of valid truncation points. Figure 4 shows distortion-rates of the remaining valid truncation points. Because $S_{j}$ is usually monotonically decreasing, it remains almost all candidate truncation points in Figure 3. Then we find a final truncation point for each block, such that the rate reaches the specified value and the distortion-rate is maximized. The number of the final truncation points is equal to the number of blocks, so there are only a little extra storage to record the truncation points.

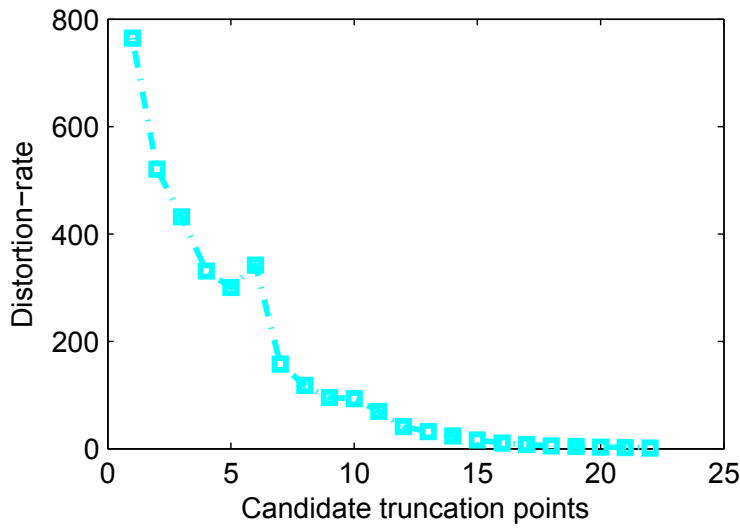

Figure 3: Distortion-rates of the candidate truncation points of the proposed method.

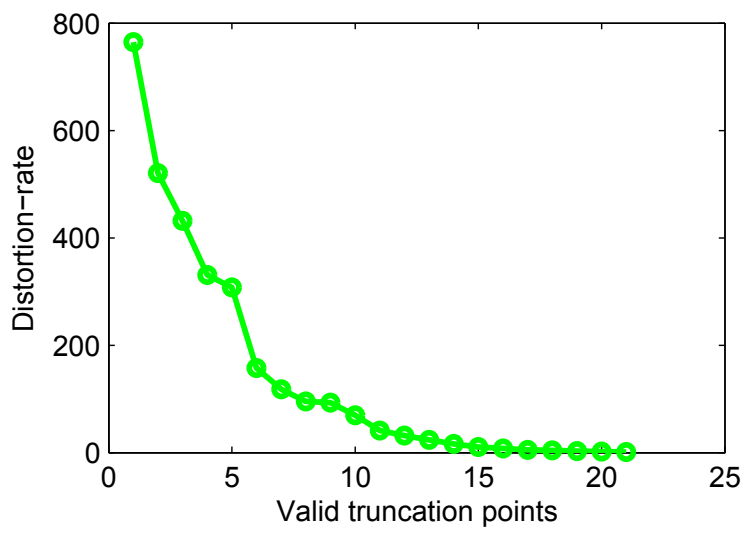

Figure 4: Distortion-rates of the valid truncation points of the proposed method. 
Suppose the blocks are of size $2^{n} \times 2^{n}$, where $N=p n$. 368 There are $2^{p} \times 2^{p}=4^{p}$ blocks. For each block, there are ${ }_{369}$ $2^{n} \times 2^{n}=2^{2 n}$ coefficients consisting the bottom level of 370 the quadtree. There are $\sum_{d=0}^{n} 4^{d}$ elements in the quadtree ${ }_{371}$ for each block, while there are $\sum_{d=0}^{N} 4^{d}$ elements in the quadtree for QCA. The memory requirement of the quadtree for a wavelet image is about $4^{p}$ times than that for a block. So, applying the rate-distortion optimization, the proposed method requires much less memory. Because the proposed method exports the code length of each block, the decoder can decode any specified blocks so that random access property is attained. As long as we give multiple bit rates and record the code length of each block at each bit rate, the proposed method can provide the quality scalability.

The proposed method is called Quadtree Coding with Adaptive scanning order and Optimized truncation, denoted by QCAO.

\subsection{A simple calculation example}

To better illustrate the proposed method, we give a simple example. Suppose that there is a $4 \times 4$ block whose coefficients are shown in the left side of Figure 1. The block is transformed into one-dimensional vector with Morton scanning order, resulting $V=$ $\{9,5,3,1 ; 4,3,2,5 ; 0,1,2,3 ; 1,0,3,4\}$. Then we can construct the quadtree according to Eq. (1) and Eq. (2), as shown in the right side of Figure 1. Table 1 shows the candidate truncation points for coding the block. We initialize the distortion by $D_{0}=\sum_{i, j} x_{i, j}^{2}=210$, the rate $R_{0}=0$ and the distortion-rate $S_{0}=$ inf. Then we first traverse the quadtree by depth-first with $T=8$. The resulting code is " 1110000000 ", and the reconstructed coefficients are $12,0,0,0,0,0,0,0,0,0,0,0,0,0,0,0$, respectively, so $R_{1}=10, D_{1}=138$ and $S_{1}=\frac{D_{1}-D_{0}}{R_{1}-R_{0}}=7.2$.

Then we traverse the quadtree from bottom level to top level with $T=4$. For each level, if the brother 375 of a coefficient is significant with $T=8$, then we 376 prefer to traverse the coefficient and its descendants, 377 i.e., with $T=4$, we perform $Q C(\Psi, 7,4), Q C(\Psi, 8,4)$, 378 $Q C(\Psi, 9,4)$ for level=2, $Q C(\Psi, 3,4), \quad Q C(\Psi, 4,4), 379$ $Q C(\Psi, 5,4)$ for level=1 in turn. After traversing with 380 $T=4$, we perform magnitude refinement pass for all 381 the previous significant coefficients, as No. 4 candidate ${ }_{382}$ truncation point in Table 1.

With $T=2$, we traverse the coefficients of No. 9, 384 $10,11,12,18,19,20$ and 4 by function $Q C$ in trun i.e., 385 we traverse the neighbors of the yellow coefficients in 386 Figure 1 first. After traversing with $T=2$, we get 10387 candidate truncation points, as shown in Table 1.

After the candidate truncation points are attained, we 389 need to calculate the valid truncation points. Because 390
$S_{4}>S_{3}$, the No. 3 candidate truncation point cannot be selected as a valid one, and we need to update $S_{4}=$ $\frac{D_{2}-D_{4}}{R_{4}-R_{2}}=\frac{102-58}{27-15}=3.7$. Because $S_{7}>S_{6}$, we need to update $S_{7}=\frac{D_{5}-D_{7}}{R_{7}-R_{5}}=\frac{16-7}{46-40}=1.5$. This process can be repeated and we can get all the valid truncation points, as shown in Table 2.

Table 1: The candidate truncation points of the proposed method for the example in Figure 1.

\begin{tabular}{cccrrc}
\hline No. & $T$ & Level & $D$ & $R$ & $S$ \\
\hline 0 & - & - & 210 & 0 & inf \\
1 & 8 & - & 138 & 10 & 7.2 \\
2 & 4 & 2 & 102 & 15 & 7.2 \\
3 & 4 & 1 & 66 & 26 & 3.3 \\
4 & 4 & - & 58 & 27 & 8.0 \\
5 & 2 & 2 & 16 & 40 & 3.2 \\
6 & 2 & 1 & 16 & 41 & 0.0 \\
7 & 2 & - & 7 & 46 & 1.8 \\
\hline
\end{tabular}

Table 2: The valid truncation points of the proposed method for the example in Figure 1.

\begin{tabular}{cccrrc}
\hline No. & $T$ & Level & $D$ & $R$ & $S$ \\
\hline 0 & - & - & 210 & 0 & inf \\
1 & 8 & - & 138 & 10 & 7.2 \\
2 & 4 & 2 & 102 & 15 & 7.2 \\
4 & 4 & - & 58 & 27 & 3.7 \\
5 & 2 & 2 & 16 & 40 & 3.2 \\
7 & 2 & - & 7 & 46 & 1.5 \\
\hline
\end{tabular}

\section{Experimental results}

In this section, to evaluate the performance of the proposed method, some experiments are conducted on three space-borne image sets: the Landsat images[29], the CCSDS image test corpus[30] and the USC-SIPI Image Database[31]. The Landsat images were produced by the Landsat-7 flight, including eight spectral bands and one panchromatic band. We select two images, i.e., Detroit and Farms, for experiment, as shown in Figure 5(a)(b). The CCSDS reference test image set includes a variety of space imaging instrument data such as solar, stellar, planetary, earth observations, etc. We select Europa3 and SpotLAb3 for experiment, as shown in Figure 5(c)(d). The USC-SIPI image database contains four volumes. The Aerials volume of the database consists of 38 images. We select Miramar NAS and Washington for experiment, as shown in Figure 5(e)(f). 


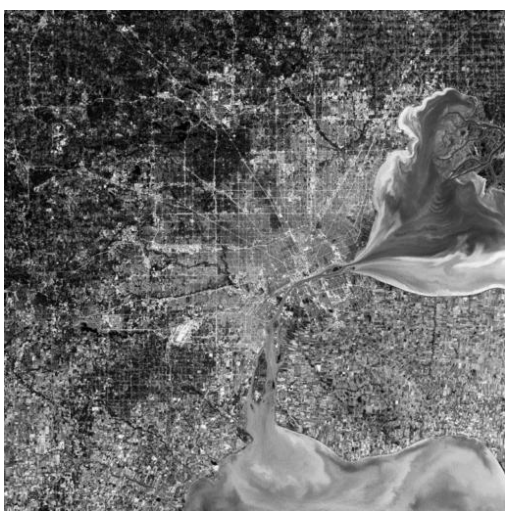

(a) Detroit

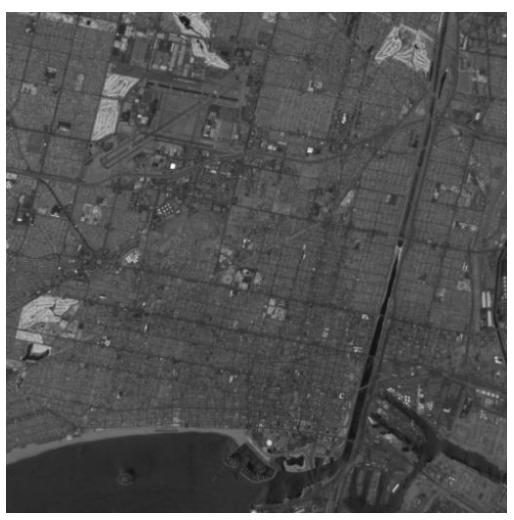

(d) SpotLAb3

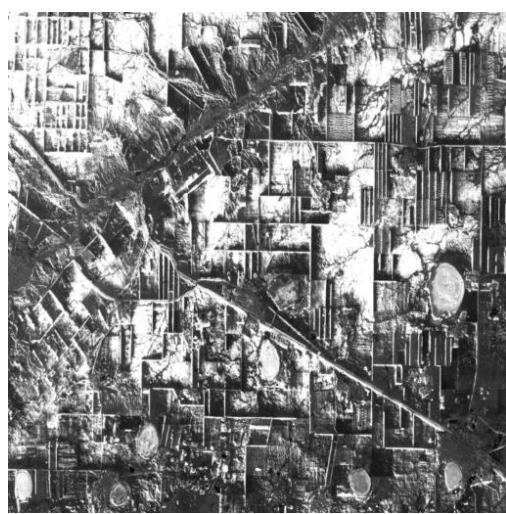

(b) Farms

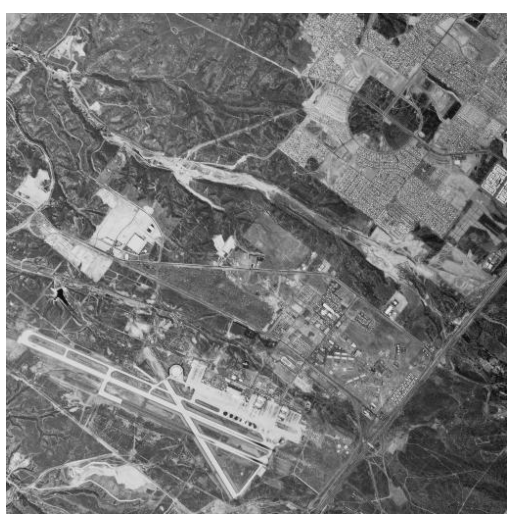

(e) MiramarNAS

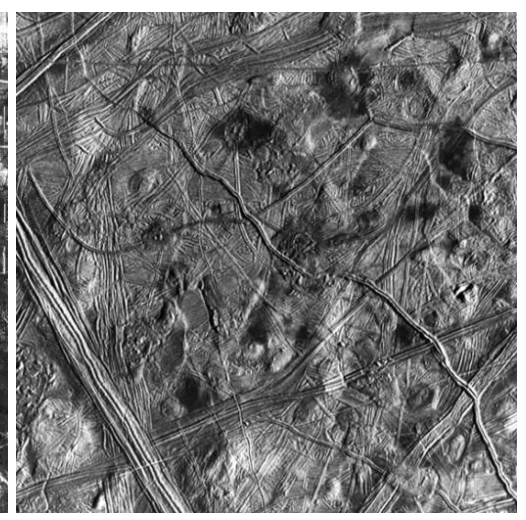

(c) Europa3

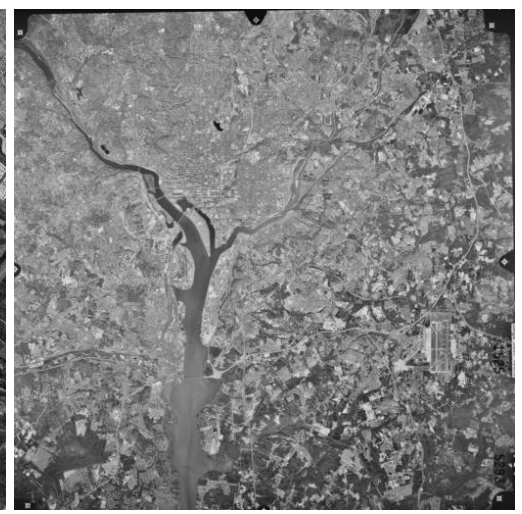

(f) Washington

Figure 5: Space-borne Images for experiment. 
Table 3: PSNRs of the proposed QCAO method and other algorithms at different bit rates for different images.

\begin{tabular}{|c|c|c|c|c|c|c|c|}
\hline \multirow[t]{2}{*}{ Image } & \multirow[t]{2}{*}{ Algorithm } & \multicolumn{6}{|c|}{ PSNR } \\
\hline & & 1bpp & $0.5 b p p$ & $0.25 b p p$ & $0.125 b p p$ & $0.0625 b p p$ & $0.0313 b p p$ \\
\hline \multirow[t]{7}{*}{ Detroit } & SPIHT & 27.27 & 24.01 & 21.78 & 20.31 & 19.07 & 17.93 \\
\hline & SPECK & 27.40 & 24.30 & 22.24 & 20.72 & 19.68 & 18.98 \\
\hline & CCSDS & 25.53 & 22.52 & 20.49 & 18.86 & 15.26 & 10.55 \\
\hline & JPEG2000 & 26.12 & 22.82 & 20.83 & 19.40 & 18.44 & 17.60 \\
\hline & BTCA & 27.59 & 24.42 & 22.30 & 20.77 & 19.72 & 18.95 \\
\hline & BTCAS & 27.50 & 24.33 & 22.33 & 20.75 & 19.73 & 18.94 \\
\hline & QCAO & 27.59 & 24.68 & 22.44 & 22.51 & 19.85 & 19.63 \\
\hline \multirow[t]{7}{*}{ Farms } & SPIHT & 26.92 & 22.80 & 20.13 & 17.99 & 16.38 & 14.71 \\
\hline & SPECK & 26.82 & 23.01 & 20.46 & 18.53 & 17.08 & 15.98 \\
\hline & CCSDS & 26.85 & 22.89 & 19.97 & 16.87 & 11.73 & 8.784 \\
\hline & JPEG2000 & 27.48 & 23.37 & 20.73 & 18.46 & 16.83 & 15.14 \\
\hline & BTCA & 26.98 & 23.10 & 20.57 & 18.58 & 17.08 & 15.92 \\
\hline & BTCAS & 26.96 & 23.11 & 20.56 & 18.58 & 17.05 & 15.90 \\
\hline & QCAO & 27.00 & 23.52 & 20.65 & 18.70 & 17.54 & 16.21 \\
\hline \multirow[t]{7}{*}{ Europa3 } & SPIHT & 26.77 & 23.09 & 20.69 & 19.01 & 17.82 & 16.57 \\
\hline & SPECK & 26.99 & 23.29 & 21.04 & 19.54 & 18.35 & 17.63 \\
\hline & CCSDS & 26.89 & 23.06 & 20.50 & 18.30 & 12.47 & 10.30 \\
\hline & JPEG2000 & 27.42 & 23.54 & 21.18 & 19.43 & 18.06 & 17.15 \\
\hline & BTCA & 27.17 & 23.47 & 21.17 & 19.58 & 18.48 & 17.61 \\
\hline & BTCAS & 27.14 & 23.47 & 21.13 & 19.56 & 18.44 & 17.59 \\
\hline & QCAO & 27.23 & 23.48 & 21.39 & 20.09 & 18.59 & 17.75 \\
\hline \multirow[t]{7}{*}{ SpotLAb3 } & SPIHT & 39.19 & 35.29 & 32.35 & 29.90 & 27.58 & 24.73 \\
\hline & SPECK & 39.24 & 35.53 & 32.72 & 30.60 & 28.97 & 27.63 \\
\hline & CCSDS & 39.34 & 35.49 & 32.55 & 29.88 & 25.54 & 13.24 \\
\hline & JPEG2000 & 39.76 & 35.67 & 32.87 & 30.51 & 28.65 & 26.68 \\
\hline & BTCA & 39.69 & 35.84 & 33.00 & 30.81 & 29.08 & 27.66 \\
\hline & BTCAS & 39.50 & 35.74 & 32.91 & 30.80 & 29.05 & 27.61 \\
\hline & QCAO & 39.60 & 35.84 & 33.21 & 30.86 & 30.08 & 27.96 \\
\hline \multirow[t]{7}{*}{ MiramarNAS } & SPIHT & 28.67 & 25.99 & 24.27 & 22.69 & 21.21 & 19.37 \\
\hline & SPECK & 28.66 & 26.12 & 24.47 & 23.09 & 21.95 & 21.01 \\
\hline & CCSDS & 28.78 & 26.20 & 24.38 & 22.38 & 14.47 & 10.75 \\
\hline & JPEG2000 & 29.28 & 26.46 & 24.57 & 22.99 & 21.70 & 20.32 \\
\hline & BTCA & 29.08 & 26.32 & 24.60 & 23.22 & 22.01 & 21.05 \\
\hline & BTCAS & 29.02 & 26.39 & 24.59 & 23.21 & 22.00 & 21.03 \\
\hline & QCAO & 29.12 & 26.40 & 24.61 & 23.30 & 22.34 & 21.14 \\
\hline \multirow[t]{7}{*}{ Washington } & SPIHT & 29.61 & 26.66 & 24.65 & 22.85 & 21.23 & 19.49 \\
\hline & SPECK & 29.69 & 26.88 & 24.91 & 23.33 & 22.06 & 21.08 \\
\hline & CCSDS & 29.75 & 26.94 & 24.73 & 22.44 & 16.65 & 10.39 \\
\hline & JPEG2000 & 30.34 & 27.19 & 24.94 & 23.23 & 21.76 & 20.45 \\
\hline & BTCA & 30.07 & 27.21 & 25.06 & 23.45 & 22.14 & 21.11 \\
\hline & BTCAS & 29.96 & 27.10 & 25.01 & 23.43 & 22.12 & 21.12 \\
\hline & QCAO & 30.09 & 27.29 & 25.50 & 23.52 & 22.63 & 21.24 \\
\hline
\end{tabular}


Table 4: SSIMs of the proposed QCAO method and other algorithms at different bit rates for different images.

\begin{tabular}{|c|c|c|c|c|c|c|c|}
\hline \multirow[t]{2}{*}{ Image } & \multirow[t]{2}{*}{ Algorithm } & \multicolumn{6}{|c|}{ SSIM } \\
\hline & & 1bpp & $0.5 b p p$ & $0.25 b p p$ & $0.125 b p p$ & $0.0625 b p p$ & $0.0313 b p p$ \\
\hline \multirow[t]{7}{*}{ Detroit } & SPIHT & 0.9755 & 0.9471 & 0.9093 & 0.8669 & 0.8145 & 0.7449 \\
\hline & SPECK & 0.9763 & 0.9507 & 0.9192 & 0.8805 & 0.8441 & 0.8097 \\
\hline & CCSDS & 0.9661 & 0.9299 & 0.8835 & 0.8186 & 0.6153 & 0.3649 \\
\hline & JPEG2000 & 0.9704 & 0.9349 & 0.8925 & 0.8453 & 0.7972 & 0.7431 \\
\hline & BTCA & 0.9773 & 0.9521 & 0.9206 & 0.8821 & 0.8459 & 0.8084 \\
\hline & BTCAS & 0.9768 & 0.9508 & 0.9196 & 0.8811 & 0.8441 & 0.8062 \\
\hline & QCAO & 0.9773 & 0.9550 & 0.9219 & 0.9240 & 0.8494 & 0.8389 \\
\hline \multirow[t]{7}{*}{ Farms } & SPIHT & 0.9862 & 0.9639 & 0.9319 & 0.8848 & 0.8249 & 0.7149 \\
\hline & SPECK & 0.9860 & 0.9657 & 0.9372 & 0.8994 & 0.8527 & 0.8034 \\
\hline & CCSDS & 0.9860 & 0.9645 & 0.9283 & 0.8407 & 0.5589 & 0.2711 \\
\hline & JPEG2000 & 0.9879 & 0.9684 & 0.9408 & 0.8957 & 0.8418 & 0.7465 \\
\hline & BTCA & 0.9865 & 0.9665 & 0.9389 & 0.9012 & 0.8536 & 0.8003 \\
\hline & BTCAS & 0.9864 & 0.9665 & 0.9387 & 0.8990 & 0.8520 & 0.7959 \\
\hline & QCAO & 0.9865 & 0.9696 & 0.9401 & 0.9023 & 0.8694 & 0.8122 \\
\hline \multirow[t]{7}{*}{ Europa3 } & SPIHT & 0.9666 & 0.9192 & 0.8536 & 0.7723 & 0.6695 & 0.5050 \\
\hline & SPECK & 0.9683 & 0.9235 & 0.8669 & 0.7995 & 0.7223 & 0.6493 \\
\hline & CCSDS & 0.9675 & 0.9184 & 0.8441 & 0.7068 & 0.3147 & 0.1817 \\
\hline & JPEG2000 & 0.9713 & 0.9277 & 0.8682 & 0.7917 & 0.6871 & 0.5832 \\
\hline & BTCA & 0.9696 & 0.9266 & 0.8712 & 0.8012 & 0.7314 & 0.6459 \\
\hline & BTCAS & 0.9694 & 0.9266 & 0.8662 & 0.8004 & 0.7211 & 0.6425 \\
\hline & QCAO & 0.9700 & 0.9268 & 0.8763 & 0.8268 & 0.7337 & 0.6614 \\
\hline \multirow[t]{7}{*}{ SpotLAb3 } & SPIHT & 0.9913 & 0.9784 & 0.9569 & 0.9221 & 0.8595 & 0.7079 \\
\hline & SPECK & 0.9917 & 0.9799 & 0.9608 & 0.9350 & 0.9016 & 0.8631 \\
\hline & CCSDS & 0.9916 & 0.9794 & 0.9588 & 0.9214 & 0.7928 & 0.2089 \\
\hline & JPEG2000 & 0.9924 & 0.9803 & 0.9619 & 0.9327 & 0.8930 & 0.8217 \\
\hline & BTCA & 0.9922 & 0.9810 & 0.9631 & 0.9380 & 0.9041 & 0.8623 \\
\hline & BTCAS & 0.9919 & 0.9806 & 0.9623 & 0.9371 & 0.9033 & 0.8597 \\
\hline & QCAO & 0.9921 & 0.9810 & 0.9649 & 0.9381 & 0.9253 & 0.8740 \\
\hline \multirow[t]{7}{*}{ MiramarNAS } & SPIHT & 0.9741 & 0.9511 & 0.9252 & 0.8896 & 0.8387 & 0.7311 \\
\hline & SPECK & 0.9741 & 0.9527 & 0.9288 & 0.9005 & 0.8658 & 0.8287 \\
\hline & CCSDS & 0.9747 & 0.9535 & 0.9270 & 0.8787 & 0.4770 & 0.1473 \\
\hline & JPEG2000 & 0.9774 & 0.9559 & 0.9305 & 0.8964 & 0.8563 & 0.7926 \\
\hline & BTCA & 0.9765 & 0.9550 & 0.9310 & 0.9037 & 0.8678 & 0.8315 \\
\hline & BTCAS & 0.9761 & 0.9551 & 0.9309 & 0.9019 & 0.8676 & 0.8281 \\
\hline & QCAO & 0.9767 & 0.9552 & 0.9312 & 0.9041 & 0.8784 & 0.8321 \\
\hline \multirow[t]{7}{*}{ Washington } & SPIHT & 0.9707 & 0.9412 & 0.9023 & 0.8460 & 0.7623 & 0.5979 \\
\hline & SPECK & 0.9714 & 0.9445 & 0.9088 & 0.8652 & 0.8092 & 0.7504 \\
\hline & CCSDS & 0.9717 & 0.9447 & 0.9041 & 0.8253 & 0.4799 & 0.1247 \\
\hline & JPEG2000 & 0.9752 & 0.9475 & 0.9091 & 0.8585 & 0.7905 & 0.6966 \\
\hline & BTCA & 0.9737 & 0.9478 & 0.9121 & 0.8692 & 0.8128 & 0.7533 \\
\hline & BTCAS & 0.9728 & 0.9462 & 0.9105 & 0.8651 & 0.8118 & 0.7469 \\
\hline & QCAO & 0.9738 & 0.9487 & 0.9212 & 0.8691 & 0.8360 & 0.7579 \\
\hline
\end{tabular}


We convert these images into gray ones and size of 418 $512 \times 512 \times 8$ before compression. In addition, the 419 9/7-tap biorthogonal wavelet filters [32] are used for 420 wavelet transform. The size of each block of the proposed QCAO method is $64 \times 64$. The Peak-Signal-to- ${ }_{421}$ Noise-Ratios (PSNR) for different Compression Ratios ${ }_{422}$ (CR) of our method are compared to those of SPIHT [2], 423 SPECK [3], CCSDS [7], JPEG2000 [12], BTCA [24] and BTCA-S [24]. The Matlab source code for BTCA 424 and QCAO are available ${ }^{1}$.

The PSNR and CR are expressed by the following 426 relations [33]:

$$
\begin{gathered}
P S N R(d B)=10 \log _{10} \frac{255^{2}}{M S E}, \\
M S E=\frac{\sum_{i=1}^{W} \sum_{j=1}^{H}\left(x_{i j}-y_{i j}\right)^{2}}{W \times H}, \\
C R(b p p)=\frac{\text { number of coded bits }}{W \times H},
\end{gathered}
$$
structed one, respectively, and the images are of size ${ }^{436}$ $W \times H$

Besides PSNR, we also use the Structural Similar- ${ }^{438}$ ity Index Measure (SSIM) to evaluate the performance. ${ }^{439}$ SSIM is correlated with the quality perception of the ${ }^{440}$ human visual system [34], which is defined as:

$$
\begin{gathered}
S S I M=l(x, y) c(x, y) s(x, y), \\
l(x, y)=\frac{2 \mu_{x} \mu_{y}+C_{1}}{\mu_{x}^{2} \mu_{y}^{2}+C_{1}}, \\
c(x, y)=\frac{2 \sigma_{x} \sigma_{y}+C_{2}}{\sigma_{x}^{2} \sigma_{y}^{2}+C_{2}}, \\
s(x, y)=\frac{\sigma_{x y}+C_{3}}{\sigma_{x} \sigma_{y}+C_{3}},
\end{gathered}
$$

where the detail parameters can be found in [34].

Table 3 and Table 4 list the PSNRs and SSIMs of the proposed method and other algorithms at six bit rates for different images, respectively. From the results, we can draw the following conclusions:

- The performance of CCSDS is the lowest, because ${ }_{458}$ it balances the complexity and PSNR.

- The performance of JPEG2000 is a much better than SPIHT or SPECK. However, JPEG2000 is too complex to be a standard for airborne mission. ke-kun-huang/
- BTCA outperforms SPIHT and SPECK, and is better than JPEG2000 at lower bit rates, which proves that BTCA is a state-of-the-art methods.

- BTCA-S sacrifices the compression ratio in order to provide position scalability, so the performance is worse than BTCA.

- The proposed QCAO achieves the highest performance. For Detroit image, the PSNR of QCAO increases an average of 1.06, 0.56, 3.91, 1.92, 0.49 and $0.52 \mathrm{~dB}$ than SPIHT, SPECK, CCSDS, JPEG2000, BTCA and BTCA-S respectively. For all the six testing image, the PSNR of QCAO increases an average of $0.96,0.38,3.28,0.62,0.24$, $0.28 \mathrm{~dB}$ than the above methods, respectively.

In order to strengthen the conclusions, we consider a larger set of test images to evaluate the performance. Here, all the 40 images from CCSDS reference testing image set are used [30]. These images include $14 \mathrm{im}$ ages with 8 bit-depth such as "costal-b1", "marstest" and "lunar", and 18 images with 10 bit-depth such as "ice-2kb1", "india-2kb1", "india-2kb4", "ocean-2kb1" , "landesV-G7-10b" and "marseille-G6-10b", 6 images with 12 bit-depth and 2 images with 16 bit-depth. Table 5 lists average PSNR and average SSIM for all the testing images of different methods at six bit rates. We can find that the mean PSNR of CCSDS is always the lowest of all methods. The proposed method is the highest of all methods at $0.5 \mathrm{bpp}, 0.25 \mathrm{bpp}, 0.125 \mathrm{bpp}, 0.0625 \mathrm{bpp}$ and $0.0313 \mathrm{bpp}$. At 1bpp, the mean PSNR of QCAO is only a little smaller than JPEG2000. Regarding the SSIM criterion, the similar results are attained. Specially, the mean SSIM of QCAO and that of JPEG2000 are equal at $1 \mathrm{bpp}$, which proves that QCAO can generate good visual effect even the PSNR is not the best.

To give a visual effect, Figure 6 shows the comparison of two compression algorithms with the image of Detroit at $0.5 \mathrm{bpp}$. We can find that the reconstructed image with QCAO is clearer than that with SPIHT. There are more textures in the reconstructed image with QCAO than those with SPIHT. Because the number of coded significant coefficients with QCAO is 12584 , while it is 11627 with SPIHT. QCAO encodes more significant coefficients than SPIHT.

Table 6 shows the encoding speed of different methods. We can find that the encoding speeds of QCAO and SPIHT are similar, and QCAO is much faster than CCSDS. Specially, the encoding process of JPEG2000

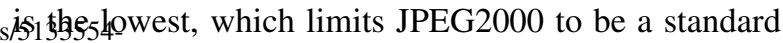
for space-borne equipments. 
Table 5: Average PSNR and average SSIM for all the images from CCSDS reference set of different methods at six bit rates.

\begin{tabular}{cccccccc}
\hline & Algorithm & 1 bpp & 0.5 bpp & 0.25 bpp & 0.125 bpp & 0.0625 bpp & 0.0313 bpp \\
\hline PSNR & SPIHT & 32.24 & 28.62 & 26.09 & 24.10 & 22.39 & 20.43 \\
& SPECK & 32.33 & 28.81 & 26.43 & 24.64 & 23.28 & 22.23 \\
& CCSDS & 32.14 & 28.56 & 25.97 & 23.49 & 18.05 & 12.59 \\
& JPEG2000 & $\mathbf{3 2 . 7 7}$ & 28.97 & 26.44 & 24.45 & 22.89 & 21.44 \\
& BTCA & 32.69 & 29.13 & 26.62 & 24.78 & 23.37 & 22.26 \\
& BTCAS & 32.56 & 29.01 & 26.56 & 24.75 & 23.33 & 22.22 \\
& QCAO & 32.71 & $\mathbf{2 9 . 1 8}$ & $\mathbf{2 6 . 7 9}$ & $\mathbf{2 5 . 1 1}$ & $\mathbf{2 3 . 8 6}$ & $\mathbf{2 2 . 5 9}$ \\
\hline SSIM & SPIHT & 0.9821 & 0.9590 & 0.9260 & 0.8805 & 0.8154 & 0.6860 \\
& SPECK & 0.9825 & 0.9611 & 0.9322 & 0.8955 & 0.8520 & 0.8050 \\
& CCSDS & 0.9814 & 0.9581 & 0.9226 & 0.8546 & 0.5894 & 0.2372 \\
& JPEG2000 & $\mathbf{0 . 9 8 3 7}$ & 0.9617 & 0.9311 & 0.8889 & 0.8340 & 0.7572 \\
& BTCA & 0.9836 & 0.9633 & 0.9349 & 0.8985 & 0.8554 & 0.8060 \\
& BTCAS & 0.9832 & 0.9624 & 0.9335 & 0.8965 & 0.8525 & 0.8018 \\
QCAO & $\mathbf{0 . 9 8 3 7}$ & $\mathbf{0 . 9 6 3 8}$ & $\mathbf{0 . 9 3 7 3}$ & $\mathbf{0 . 9 0 6 8}$ & $\mathbf{0 . 8 6 7 5}$ & $\mathbf{0 . 8 2 0 7}$ \\
\hline
\end{tabular}

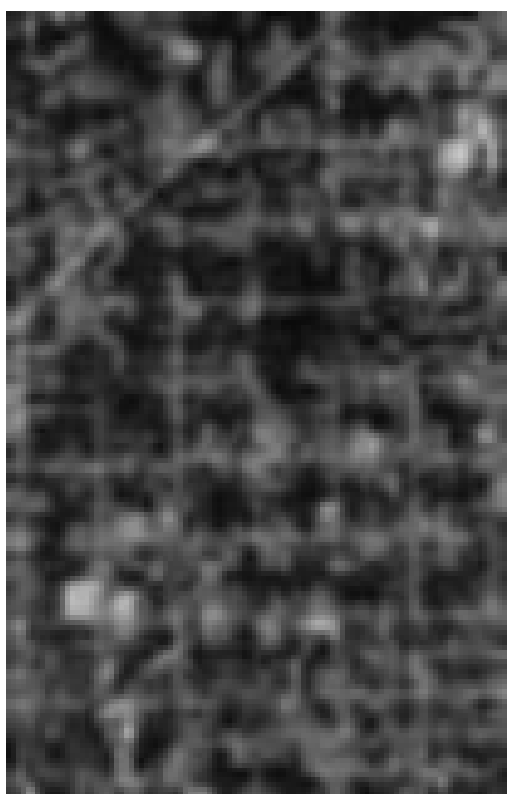

(a) Part of original "Detroit" image

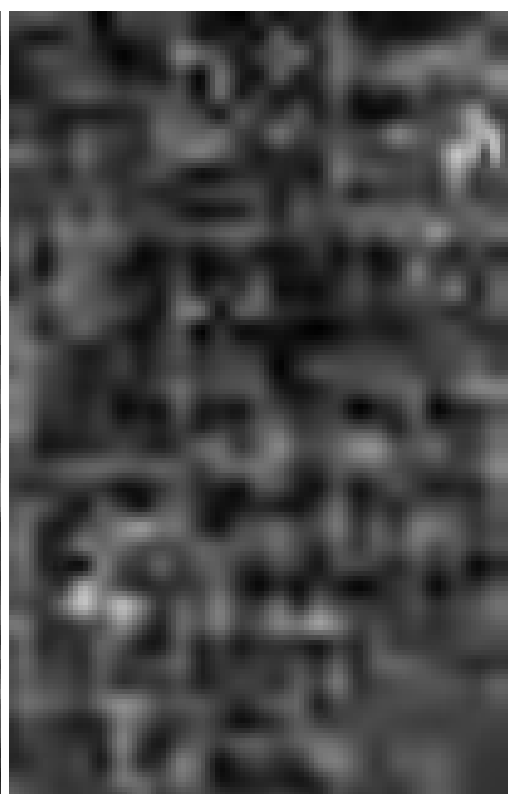

(b) Reconstructed image with QCAO

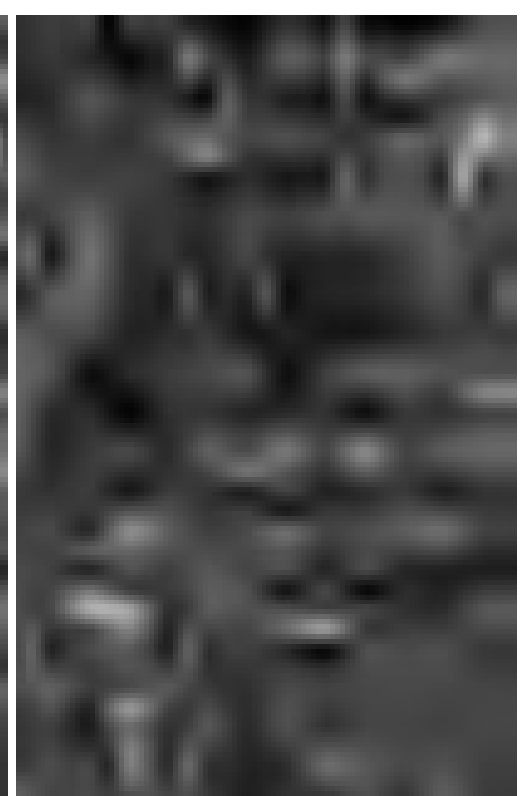

(c) Reconstructed image with SPIHT

Figure 6: Comparison of two compression algorithms with the image of Detroit at $0.5 \mathrm{bpp}$. The reconstructed image with QCAO is clearer than that with SPIHT. There are more textures in the reconstructed image with QCAO than those with SPIHT. 
Table 6: The encoding speed for different methods.

\begin{tabular}{llcl}
\hline Methods & \multicolumn{3}{c}{ Coding time (s) } \\
\cline { 2 - 4 } & 0.25 & 0.50 & 1.00 \\
\hline SPIHT & 0.09 & 0.10 & 0.12 \\
SPECK & 0.10 & 0.12 & 0.14 \\
CCSDS & 0.28 & 0.75 & 1.56 \\
JPEG2000 & 0.34 & 0.95 & 1.99 \\
BCTA & 0.16 & 0.18 & 0.21 \\
BCTAS & 0.17 & 0.19 & 0.23 \\
QCAO & 0.15 & 0.16 & 0.19 \\
\hline
\end{tabular}

\section{Conclusion}

In this paper, an efficient compression method is proposed for space-borne image. We first propose an adap- ${ }^{529}$ tive scanning order for quadtree coding, then we divide the entire wavelet image to several blocks and encode ${ }_{532}$ them individually. To control bit rate for each block, ${ }_{53}$ we set the points exactly after coding each level of the ${ }^{534}$ quadtree as the candidate truncation points. The pro- ${ }^{535}$ posed method gets higher compression ratio, less memory requirement and fast encoding speed.

\section{References}

[1] J. M. Shapiro, Embedded image coding using zerotrees of 543 wavelet coefficients, IEEE Transations on Signal Processing 544 41 (12) (1993) 3445-3462.

[2] A. Said, W. A. Pearlman, A new, fast, and efficient image codec based on set partitioning in hierarchical trees, IEEE Transactions on Circuits and Systems for Video Technology 6 (3) (1996) 243-250.

[3] W. A. Pearlman, A. Islam, N. Nagaraj, A. Said, Efficient, low-complexity image coding with a set-partitioning embedded 551 block coder, IEEE Transactions on Circuits and Systems for ${ }^{552}$ Video Technology 14 (11) (2004) 1219-1235.

[4] P. Strobach, Quadtree-structured recursive plane decomposition coding of images, IEEE Transactions on Signal Processing 39 (6) (1991) 1380-1397.

[5] R. L. Joshi, V. J. Crump, T. R. Fischer, Image subband coding using arithmetic coded trellis coded quantization, IEEE Transactions on Circuits and Systems for Video Technology 5 (6) (1995) 515523.

[6] A. Munteanu, J. Cornelis, G. V. D. Auwera, P. Cristea, Wavelet image compression - the quadtree coding approach, IEEE Transactions on Information Technology in Biomedicine 3 (3) (1999) 176-185.

[7] [Online], Image data compression, Nov. ${ }^{565}$ 2005. ser. blue book, CCSDS 122.0-b-1, http: / / public.ccsds.org/publications/BlueBooks

[8] F. Garcia-Vilchez, J. Serra-Sagrista, Extending the CCSDS rec- ${ }^{568}$ ommendation for image data compression for remote sensing 569 scenarios, IEEE Transactions on Geoscience and Remote Sens- 570 ing 47 (10) (2009) 3431-3445.

[9] X. S. Hou, J. Yang, G. F. Jiang, X. M. Qian, Complex sar image 5 compression based on directional lifting wavelet transform with high clustering capability, IEEE Transactions on Geoscience and Remote Sensing 51 (1) (2013) 527-538.

[10] D. Taubman, High performance scalable image compression with EBCOT, IEEE Transations on Image Processing 9 (7) (2000) 1158-1170

[11] B. Li, R. Yang, H. X. Jiang, Remote-sensing image compression using two-dimensional oriented wavelet transform, IEEE Transactions on Geoscience and Remote Sensing 49 (1) (2011) 236-250.

[12] P. Kulkarni, A. Bilgin, M. W. Marcellin, J. C. Dagher, J. H. Kasner, T. J. Flohr, J. C. Rountree, Hyperspectral Data Compression, Springer US, Boston, MA, 2006, Ch. Compression of Earth Science Data with JPEG2000, pp. 347-378.

[13] H. ZainEldin, M. A. Elhosseini, H. A. Ali, A modified listless strip based SPIHT for wireless multimedia sensor networks, Computers and Electrical Engineeringdoi:http://dx.doi.org/10.1016/j.compeleceng.2015.10.001.

[14] V. B. Raju, K. J. Sankar, C. D. Naidu, S. Bachu, Multispectral image compression for various band images with high resolution improved DWT SPIHT, International Journal of Signal Processing, Image Processing and Pattern Recognition 9 (2) (2016) 271-286.

[15] M. Cagnazzo, L. Cicala, G. Poggi, L. Verdoliva, Lowcomplexity compression of multispectral images based on classified transform coding, Signal Processing: Image Communication 21 (10) (2006) $850-861$.

[16] M. Cagnazzo, S. Parrilli, G. Poggi, L. Verdoliva, Improved class-based coding of multispectral images with shape-adaptive wavelet transform, IEEE Geoscience and Remote Sensing Letters 4 (4) (2007) 566-570.

[17] G. Gelli, G. Poggi, Compression of multispectral images by spectral classification and transform coding., IEEE Transactions on Image Processing 8 (4) (1999) 476-489.

[18] L. Wang, J. Bai, J. J. Wu, G. Jeon, Hyperspectral image compression based on lapped transform and tucker decomposition, Signal Processing: Image Communication 36 (8) (2015) 63 69.

[19] L. Zhang, L. Zhang, D. Tao, X. Huang, B. Du, Compression of hyperspectral remote sensing images by tensor approach, Neurocomputing 147 (2015) $358-363$

[20] M. Cagnazzo, G. Poggi, L. Verdoliva, Region-based transform coding of multispectral images, IEEE Transactions on Image Processing 16 (12) (2008) 2916-2926.

[21] I. Blanes, E. Magli, J. Serra-Sagrista, A tutorial on image compression for optical space imaging systems, IEEE Geoscience and Remote Sensing Magazine 2 (3) (2014) 8-26.

[22] N. Amrani, J. Serra-Sagrist, V. Laparra, M. W. Marcellin, Regression wavelet analysis for lossless coding of remote-sensing data, IEEE Transactions on Geoscience and Remote Sensing 54 (9) (2016) 1-12.

[23] X. S. Hou, M. Han, C. Gong, X. M. Qian, SAR complex image data compression based on quadtree and zerotree coding in discrete wavelet transform domain: A comparative study, Neurocomputing 148 (2015) $561-568$.

[24] K. K. Huang, D. Q. Dai, A new on-board image codec based on binary tree with adaptive scanning order in scan-based mode, IEEE Transactions on Geoscience and Remote Sensing 50 (10) (2012) 3737-3750.

[25] C. P. Shi, J. P. Zhang, Y. Zhang, A novel vision-based adaptive scanning for the compression of remote sensing images, IEEE Transactions on Geoscience and Remote Sensing 54 (3) (2016) 1336-1348.

[26] C. P. Shi, J. P. Zhang, Y. Zhang, Content-based onboard compression for remote sensing images, Neurocomputing 191 (2016) 330-340. 
[27] A. Fiengo, G. Chierchia, M. Cagnazzo, B. Pesquet-Popescu, Rate allocation in predictive video coding using a convex optimization framework, IEEE Transaction on Image Processing 26 (1) (2017) 479-489.

[28] C. Greco, I. D. Nemoianu, M. Cagnazzo, B. Pesquet-Popescu, Rate-distortion-optimized multi-view streaming in wireless environment using network coding, EURASIP Journal on Advances in Signal Processing 2016 (1) (2016) 17.

[29] [Online], United states geological survey, landsat project website, http://landsat.usgs.gov/.

[30] [Online], Consultative committee for space data systems, CCSDS image test, http://cwe.ccsds.org/sls/docs/sls-dc/.

[31] [Online], The USC-SIPI image database, http://sipi.usc.edu/database/.

[32] M. Antonini, M. Barlaud, P. Mathieu, I. Daubechies, Image coding using wavelet transform, IEEE Transations on Image Processing 1 (2) (1992) 205-220.

[33] D. J. Granrath, The role of human visual models in image processing, Proceedings of the IEEE 69 (5) (1981) 552-561.

[34] Z. Wang, A. C. Bovik, H. R. Sheikh, E. P. Simoncelli, Image quality assessment: from error visibility to structural similarity, IEEE Transactions on Image Processing 8 (03) (2004) 600-612. 